\title{
EXISTENCE AND GLOBAL STABILITY OF POSITIVE PERIODIC SOLUTIONS OF A DISCRETE DELAY COMPETITION SYSTEM
}

\author{
HAI-FENG HUO and WAN-TONG LI
}

\author{
Received 15 October 2002
}

\begin{abstract}
The existence and the global stability of positive periodic solutions of a discrete competition model is studied. The model incorporates time delays and allows for a fluctuating environment. By means of some standard procedures of the topological degree method and the construction of a suitable Lyapunov function, sufficient conditions are obtained to ensure the existence and the global stability of positive periodic solutions of the above systems.
\end{abstract}

2000 Mathematics Subject Classification: 92D25, 39A10.

1. Introduction. There has been quite a lot of excellent work on the study of ecological competition systems (see $[3,4,11,16]$ and the references therein). The classical two-species Lotka-Volterra autonomous competition model is described by the following systems of differential equations:

$$
\begin{aligned}
& u^{\prime}(t)=u(t)\left[r_{1}-a u(t)-b v(t)\right], \\
& v^{\prime}(t)=v(t)\left[r_{2}-c u(t)-d v(t)\right],
\end{aligned}
$$

Though much progress has been seen in the competition systems (1.1), such systems are not well studied in the sense that most results are continuous time cases related. However, many authors [1, 2, 9, 17] have argued that the discrete time models governed by difference equations are more appropriate than the continuous ones when the populations have nonoverlapping generations. Discrete time models can also provide efficient computational models of continuous models for numerical simulations. In [20], Saito et al. considered the following discrete competition system with constant delays $n_{1}, n_{2}, l_{1}$, and $l_{2}$ :

$$
\begin{aligned}
& u(k+1)=u(k) \exp \left[r_{1}-a u\left(k-n_{1}\right)-b v\left(k-n_{2}\right)\right], \\
& v(k+1)=v(k) \exp \left[r_{2}-c u\left(k-l_{1}\right)-d v\left(k-l_{2}\right)\right],
\end{aligned}
$$

and they obtained a necessary and sufficient condition for the permanence of (1.2). On the other hand, the variation of the environment plays an important 
role in many biological and ecological systems. In particular, the effects of a periodically varying environment are important for the evolutionary theory as the selective forces on systems in a fluctuating environment differ from those in a stable environment. Thus, the assumption of periodicity of the parameters (in a way) incorporates the periodicity of the environment (e.g., seasonal effects of weather, food supplies, mating habits, etc.). In fact, it has been suggested by Nicholson [18] that any periodic change of climate tends to impose its period upon oscillations of internal origin or to cause such oscillations to have a harmonic relation to periodic climatic changes. Therefore, it is physically reasonable to assume that parameters in the models are periodic functions. We remark that in recent years periodic population dynamics has become a very popular subject. In fact, several different periodic models have been studied in $[14,15,21,22,23,25]$.

Motivated by all of the above, the principal aim of this paper is to study the global existence of periodic solutions of the following discrete delay competition system:

$$
\begin{aligned}
& u(k+1)=u(k) \exp \left[r_{1}(k)-a(k) u\left(k-n_{1}\right)-b(k) v\left(k-n_{2}\right)\right], \\
& v(k+1)=v(k) \exp \left[r_{2}(k)-c(k) u\left(k-l_{1}\right)-d(k) v\left(k-l_{2}\right)\right],
\end{aligned}
$$

where $u(k), v(k)$ are the population sizes of the two competitors at generation $k, r_{i}(k), i=1,2$, is the growth rate of population $i$ at $k$ th generation, $a(k)$, $d(k)$ measure the intensity of intraspecific competition of two species, and $b(k), c(k)$ measure the intensity of interspecific competition of two species. It is assumed that $r_{i}(k), i=1,2, a(k), b(k), c(k), d(k): \mathbb{Z} \rightarrow \mathbb{R}^{+}$are $\omega$ periodic, that is,

$$
\begin{gathered}
r_{i}(k+\omega)=r_{i}(k), \quad i=1,2, \quad c(k+\omega)=c(k), \\
b(k+\omega)=b(k), \quad a(k+\omega)=a(k), \quad d(k+\omega)=d(k),
\end{gathered}
$$

for any $k \in \mathbb{Z}, n_{i}, l_{i}$ are nonnegative integers. For biological reasons, we only consider the following initial condition:

$$
\begin{aligned}
& u(-m) \geq 0, \quad m=1, \ldots, \max \left\{n_{1}, l_{1}\right\}, u(0)>0, \\
& v(-m) \geq 0, \quad m=1, \ldots, \max \left\{n_{2}, l_{2}\right\}, v(0)>0 .
\end{aligned}
$$

The method used here will be the coincidence degree theory developed by Gaines and Mawhin [10]. Such an approach was adopted in [5, 13, 14, 15, 23].

In mathematical biology, the global stability of a population model is of great interest. There is extensive literature related to this topic for differential equation models (see [16, 19, 24] and the references therein). In contrast, 
the studies for many-species difference models focus their attention on the permanence of models or extinction of populations (see $[6,7,8,12,20]$ and the references therein). Few papers investigate the global stability of positive periodic solutions of these models. So the second purpose of this paper is to study the global stability of the positive periodic solution of system (1.3).

The organization of this paper is the following. In Section 2, we obtain the sufficient conditions to ensure the existence of positive periodic solution of system (1.3). In Section 3, we study the global stability of the positive periodic solution of system (1.3).

2. Existence of positive periodic solutions. In this section, we use the Mawhin's continuation theorem of the coincidence degree theory to investigate the existence of at least one positive periodic solution of system (1.3).

Let $X, Y$ be real Banach spaces, $L: \operatorname{Dom} L \subset X \rightarrow Y$ a Fredholm mapping of index zero (index $L=\operatorname{dim} \operatorname{ker} L-\operatorname{codim} \operatorname{Im} L$ ), and $P: X \rightarrow X, Q: Y \rightarrow Y$ continuous projectors such that $\operatorname{Im} P=\operatorname{Ker} L, \operatorname{Ker} Q=\operatorname{Im} L, X=\operatorname{Ker} L \oplus \operatorname{Ker} P$, and $Y=\operatorname{Im} L \oplus \operatorname{Im} Q$. Denote by $L_{p}$ the restriction of $L$ to $\operatorname{Dom} L \cap \operatorname{Ker} P, K_{p}$ : $\operatorname{Im} L \rightarrow \operatorname{Ker} P \cap \operatorname{Dom} L$ the inverse (to $L_{p}$ ), and $J: \operatorname{Im} Q \rightarrow \operatorname{Ker} L$ an isomorphism of $\operatorname{Im} Q$ onto $\operatorname{Ker} L$. For convenience, we introduce a continuation theorem [10, page 40] as follows.

LEMMA 2.1. Let $\Omega \subset X$ be an open bounded set, $N: X \rightarrow Y$ a continuous operator which is L-compact on $\bar{\Omega}$ (i.e., $Q N: \bar{\Omega} \rightarrow Y$ and $K_{p}(I-Q) N: \bar{\Omega} \rightarrow Y$ are compact), and $L: \operatorname{Dom} L \subset X \rightarrow Y$ a Fredholm mapping of index zero with $\Omega$ open bounded in $X$. Assume that

(a) for each $\lambda \in(0,1), x \in \partial \Omega \cap \operatorname{Dom} L$,

$$
L x \neq \lambda N x
$$

(b) for each $x \in \partial \Omega \cap \operatorname{Ker} L$,

$$
\begin{gathered}
Q N x \neq 0, \\
\operatorname{deg}\{J Q N x, \Omega \cap \operatorname{Ker} L, 0\} \neq 0 .
\end{gathered}
$$

Then $L x=N x$ has at least one solution in $\bar{\Omega} \cap \operatorname{Dom} L$.

For convenience, we will introduce the notations

$$
I_{\omega}=\{0,1, \ldots, \omega-1\}, \quad \bar{u}=\frac{1}{\omega} \sum_{k=0}^{\omega-1} u(k), \quad(u)_{M}=\max _{k \in I_{\omega}} u(k),
$$

where $\{u(k)\}$ is an $\omega$-periodic sequence of real numbers defined for $k \in \mathbb{Z}$ and $\omega$ is a fixed positive integer. 
LEMMA 2.2 [5]. Let $u: \mathbb{Z} \rightarrow \mathbb{R}$ be $\omega$ periodic, that is, $u(k+\omega)=u(k)$. Then, for any fixed $k_{1}, k_{2} \in I_{\omega}$, and for any $k \in \mathbb{Z}$,

$$
\begin{aligned}
& u(k) \leq u\left(k_{1}\right)+\sum_{s=0}^{\omega-1}|u(s+1)-u(s)|, \\
& u(k) \geq u\left(k_{2}\right)-\sum_{s=0}^{\omega-1}|u(s+1)-u(s)| .
\end{aligned}
$$

Define

$$
l_{2}=\left\{x=\{x(k)\}: x(k) \in \mathbb{R}^{2}, k \in \mathbb{Z}\right\} .
$$

For $a=\left(a_{1}, a_{2}\right)^{T} \in \mathbb{R}^{2}$, define $|a|=\max \left\{a_{1}, a_{2}\right\}$. Let $l^{\omega} \subset l_{2}$ denote the subspace of all $\omega$ periodic sequences equipped with the usual supremum norm $\|\cdot\|$, that is,

$$
\|x\|=\max _{k \in I_{\omega}}|x(k)|, \quad \text { for any } x=\{x(k): k \in \mathbb{Z}\} \in l^{\omega} .
$$

We can show that $l^{\omega}$ is a finite-dimensional Banach space.

Let

$$
\begin{aligned}
& l_{0}^{\omega}=\left\{x=\{x(k)\} \in l^{\omega}: \sum_{k=0}^{\omega-1} x(k)=0\right\}, \\
& l_{c}^{\omega}=\left\{x=\{x(k)\} \in l^{\omega}: x(k)=h \in \mathbb{R}^{2}, k \in \mathbb{Z}\right\},
\end{aligned}
$$

then it follows that $l_{0}^{\omega}$ and $l_{c}^{\omega}$ are both closed linear subspaces of $l^{\omega}$ and

$$
l^{\omega}=l_{0}^{\omega} \oplus l_{c}^{\omega}, \quad \operatorname{dim} l_{c}^{\omega}=2 .
$$

Now we state our fundamental theorem about the existence of a positive $\omega$-periodic solution of system (1.3).

THEOREM 2.3. Assume that the following conditions hold:

(i) $\bar{r}_{1}-\bar{b}\left(\bar{r}_{1} / \bar{a}\right) \exp 2 \bar{r}_{2} \omega>0$;

(ii) $\bar{r}_{2}-\bar{c}\left(\bar{r}_{1} / \bar{b}\right) \exp 2 \bar{r}_{1} \omega>0$;

(iii) the system of equations

$$
\bar{r}_{1}-\bar{a} v_{1}-\bar{b} v_{2}=0, \quad \bar{r}_{2}-\bar{c} v_{1}-\bar{d} v_{2}=0,
$$

has a unique positive solution $\left(v_{1}, v_{2}\right) \in \mathbb{R}^{2}$.

Then system (1.3) has at least one positive $\omega$-periodic solution, say $x^{*}(k)=$ $\left\{u^{*}(k), v^{*}(k)\right\}$ and there exist positive constants $\alpha_{i}$ and $\beta_{i}, i=1,2$, such that

$$
\alpha_{1} \leq u^{*}(k) \leq \beta_{1}, \quad \alpha_{2} \leq v^{*}(k) \leq \beta_{2}, \quad k \in \mathbb{Z} .
$$


Proof. Let

$$
u(k)=\exp \left\{x_{1}(k)\right\}, \quad v(k)=\exp \left\{x_{2}(k)\right\}
$$

Consider the system

$$
\begin{aligned}
& x_{1}(k+1)-x_{1}(k)=r_{1}(k)-a(k) \exp x_{1}\left(k-n_{1}\right)-b(k) \exp x_{2}\left(k-n_{2}\right), \\
& x_{2}(k+1)-x_{2}(k)=r_{2}(k)-c(k) \exp x_{1}\left(k-l_{1}\right)-d(k) \exp x_{2}\left(k-l_{2}\right) .
\end{aligned}
$$

In order to apply Lemma 2.1 to system (2.12), we take $X=Y=l^{\omega},(L x)(k)=$ $x(k+1)-x(k)$, and

$$
(N x)(k)=\left[\begin{array}{c}
r_{1}(k)-a(k) \exp x_{1}\left(k-n_{1}\right)-b(k) \exp x_{2}\left(k-n_{2}\right) \\
r_{2}(k)-c(k) \exp x_{1}\left(k-l_{1}\right)-d(k) \exp x_{2}\left(k-l_{2}\right)
\end{array}\right],
$$

for any $x \in X$ and $k \in \mathbb{Z}$. It is trivial to see that $L$ is a bounded linear operator and

$$
\operatorname{Ker} L=l_{c}^{\omega}, \quad \operatorname{Im} L=l_{0}^{\omega},
$$

as well as

$$
\operatorname{dim} \operatorname{Ker} L=2=\operatorname{codim} \operatorname{Im} L,
$$

then it follows that $L$ is a Fredholm mapping of index zero.

Define

$$
P x=\frac{1}{\omega} \sum_{s=0}^{\omega-1} x(s), \quad x \in X, \quad Q z=\frac{1}{\omega} \sum_{s=0}^{\omega-1} z(s), \quad z \in Y .
$$

It is not difficult to show that $P$ and $Q$ are continuous projectors such that

$$
\operatorname{Im} P=\operatorname{Ker} L, \quad \operatorname{Im} L=\operatorname{Ker} Q=\operatorname{Im}(I-Q) .
$$

Furthermore, the generalized inverse (to $L$ ) $K_{p}: \operatorname{Im} L \rightarrow \operatorname{Ker} P \cap \operatorname{dom} L$ has the form

$$
K_{p}(z)=\sum_{s=0}^{k-1} z(s)-\frac{1}{\omega} \sum_{s=0}^{\omega-1}(\omega-s) z(s)
$$

By the Lebesgue theorem, we can prove that $Q N$ and $K_{p}(I-Q) N$ are continuous. Since $X$ is a finite-dimensional Banach space, using the Arzela-Ascoli 
theorem, we can also show that $Q N(\bar{\Omega})$ and $K_{p}(I-Q) N(\bar{\Omega})$ are relatively compact for any open bounded set $\Omega \subset X$. Hence, $N$ is $L$-compact on $\bar{\Omega}$, here $\Omega$ is any open bounded set in $X$.

Now we reach the position to search for an appropriate open bounded subset $\Omega$ for the application of Lemma 2.1. Corresponding to the equation $L x=\lambda N x$, $\lambda \in(0,1)$, we have

$$
\begin{aligned}
x_{1}(k+1)-x_{1}(k)= & \lambda\left[r_{1}(k)-a(k) \exp x_{1}\left(k-n_{1}\right)\right. \\
& \left.-b(k) \exp x_{2}\left(k-n_{2}\right)\right], \\
x_{2}(k+1)-x_{2}(k)= & \lambda\left[r_{2}(k)-c(k) \exp x_{1}\left(k-l_{1}\right)\right. \\
& \left.-d(k) \exp x_{2}\left(k-l_{2}\right)\right] .
\end{aligned}
$$

Suppose that $x(k)=\left(x_{1}(k), x_{2}(k)\right) \in X$ is a solution of system (2.12) for a certain $\lambda \in(0,1)$. Summing up both sides of (2.19) from 0 to $\omega-1$, we obtain

$$
\begin{aligned}
& \sum_{k=0}^{\omega-1}\left[r_{1}(k)-a(k) \exp x_{1}\left(k-n_{1}\right)-b(k) \exp x_{2}\left(k-n_{2}\right)\right] \\
& \quad=\sum_{k=0}^{\omega-1}\left[x_{1}(k+1)-x_{1}(k)\right]=0 \\
& \sum_{k=0}^{\omega-1}\left[r_{2}(k)-c(k) \exp x_{1}\left(k-l_{1}\right)-d(k) \exp x_{2}\left(k-l_{2}\right)\right] \\
& =\sum_{k=0}^{\omega-1}\left[x_{2}(k+1)-x_{2}(k)\right]=0 .
\end{aligned}
$$

Thus

$$
\begin{gathered}
\sum_{k=0}^{\omega-1}\left[a(k) \exp x_{1}\left(k-n_{1}\right)+b(k) \exp x_{2}\left(k-n_{2}\right)\right]=\bar{r}_{1} \omega, \\
\sum_{k=0}^{\omega-1}\left[c(k) \exp x_{1}\left(k-l_{1}\right)+d(k) \exp x_{2}\left(k-l_{2}\right)\right]=\bar{r}_{2} \omega .
\end{gathered}
$$

From (2.19), (2.21), and (2.22), it follows that

$$
\begin{aligned}
\sum_{k=0}^{\omega-1}[ & \left.x_{1}(k+1)-x_{1}(k)\right] \\
& =\lambda \sum_{k=0}^{\omega-1}\left[r_{1}(k)-a(k) \exp x_{1}\left(k-n_{1}\right)-b(k) \exp x_{2}\left(k-n_{2}\right)\right] \\
& <\bar{r}_{1} \omega+\sum_{k=0}^{\omega-1}\left[a(k) \exp x_{1}\left(k-n_{1}\right)+b(k) \exp x_{2}\left(k-n_{2}\right)\right] \\
& =2 \bar{r}_{1} \omega,
\end{aligned}
$$




$$
\begin{aligned}
\sum_{k=0}^{\omega-1}[ & \left.x_{2}(k+1)-x_{2}(k)\right] \\
& =\lambda \sum_{k=0}^{\omega-1}\left[r_{2}(k)-c(k) \exp x_{1}\left(k-l_{1}\right)-d(k) \exp x_{2}\left(k-l_{2}\right)\right] \\
& <\bar{r}_{2} \omega+\sum_{k=0}^{\omega-1}\left[c(k) \exp x_{1}\left(k-l_{1}\right)+d(k) \exp x_{2}\left(k-l_{2}\right)\right] \\
& =2 \bar{r}_{2} \omega .
\end{aligned}
$$

That is

$$
\begin{aligned}
& \sum_{k=0}^{\omega-1}\left[x_{1}(k+1)-x_{1}(k)\right]<2 \bar{r}_{1} \omega, \\
& \sum_{k=0}^{\omega-1}\left[x_{2}(k+1)-x_{2}(k)\right]<2 \bar{r}_{2} \omega .
\end{aligned}
$$

Note that $\left(x_{1}(k), x_{2}(k)\right)^{T} \in X$, then there exists $\xi_{i}, \eta_{i} \in I_{\omega}, i=1,2,3$, such that

$$
x_{i}\left(\xi_{i}\right)=\min _{k \in I_{\omega}} x_{i}(k), \quad x_{i}\left(\eta_{i}\right)=\max _{k \in I_{\omega}} x_{i}(k), \quad i=1,2 .
$$

From (2.21), (2.22), and (2.25), we have

$$
x_{2}\left(\xi_{2}\right)<\ln \left(\frac{\bar{r}_{1}}{\bar{b}}\right), \quad x_{1}\left(\xi_{1}\right)<\ln \left(\frac{\bar{r}_{1}}{\bar{a}}\right) .
$$

Then, by (2.26) and Lemma 2.2, we have

$$
\begin{aligned}
& x_{1}(k) \leq x_{1}\left(\xi_{1}\right)+\sum_{s=0}^{\omega-1}\left[x_{1}(s+1)-x_{1}(s)\right]<\ln \left(\frac{\bar{r}_{1}}{\bar{b}}\right)+2 \bar{r}_{1} \omega:=M_{1}, \\
& x_{2}(k) \leq x_{2}\left(\xi_{1}\right)+\sum_{s=0}^{\omega-1}\left[x_{2}(s+1)-x_{2}(s)\right]<\ln \left(\frac{\bar{r}_{1}}{\bar{a}}\right)+2 \bar{r}_{2} \omega:=M_{2} .
\end{aligned}
$$

By virtue of (2.21) and assumption (i), we obtain that

$$
\begin{aligned}
\sum_{k=0}^{\omega-1}\left[a(k) \exp x_{1}\left(k-n_{1}\right)\right] & =\bar{r}_{1} \omega-\sum_{k=0}^{\omega-1}\left[b(k) \exp x_{2}\left(k-n_{2}\right)\right] \\
& >\bar{r}_{1} \omega-\bar{b} \omega \frac{\bar{r}_{1}}{\bar{a}} \exp 2 \bar{r}_{2} \omega>0
\end{aligned}
$$

which implies that there exist a point $k_{1} \in I_{\omega}$ and a constant $M_{3}>0$ such that $x\left(k_{1}-n_{1}\right)>-M_{3}$, denote $k_{1}-n_{1}=k_{1}^{*}+m_{1} \omega, k_{1}^{*} \in I_{\omega}, m_{1}$ is an integer, then

$$
x_{1}\left(k_{1}^{*}\right)>-M_{3}
$$


Similarly, from (2.22) and assumption (ii), we also obtain that there exist a point $k_{2}^{*} \in[0, \omega]$ and a constant $M_{4}>0$ such that

$$
x_{2}\left(k_{2}^{*}\right)>-M_{4}
$$

By (2.24), (2.29), (2.30), and Lemma 2.2, it is easy to see that

$$
x_{1}(k)>-\left(M_{3}+2 \bar{r}_{1} \omega\right), \quad x_{2}(k)>-\left(M_{4}+2 \bar{r}_{2} \omega\right) .
$$

It follows from (2.27) and (2.31) that

$$
\begin{aligned}
& \left|x_{1}(k)\right|<\max \left\{M_{1}, M_{3}+2 \bar{r}_{1} \omega\right\}=M_{5}, \\
& \left|x_{2}(k)\right|<\max \left\{M_{2}, M_{4}+2 \bar{r}_{2} \omega\right\}=M_{6} .
\end{aligned}
$$

Clearly, $M_{i}(i=1,2,3,4,5,6)$ are independent of $\lambda$, and under assumption (iii) of the theorem, the system of the algebraic equations

$$
\bar{r}_{1}-\bar{a} v_{1}-\bar{b} v_{2}=0, \quad \bar{r}_{2}-\bar{c} v_{1}-\bar{d} v_{2}=0,
$$

has a unique solution $\left(v_{1}^{*}, v_{2}^{*}\right)$ which satisfies $v_{1}^{*}>0$ and $v_{2}^{*}>0$. Denote

$$
M=M_{5}+M_{6}+K,
$$

where $K>0$ is taken sufficiently large so that the unique solution of system (2.33) satisfies

$$
\left\|\left(v_{1}^{*}, v_{2}^{*}\right)\right\|=\left|v_{1}^{*}\right|+\left|v_{2}^{*}\right|<M .
$$

Now we take

$$
\Omega=\left\{x=\left(x_{1}(k), x_{2}(k)\right)^{T} \in X:\left\|\left(x_{1}, x_{2}\right)\right\|<M\right\} .
$$

This satisfies condition (a) of Lemma 2.1. When

$$
x \in \partial \Omega \cap \operatorname{Ker} L=\partial \Omega \cap \mathbb{R}^{2},
$$

$x$ is a constant vector in $\mathbb{R}^{2}$ with $\|x\|=M$. Then

$$
Q N\left[\begin{array}{l}
x_{1} \\
x_{2}
\end{array}\right]=\left[\begin{array}{l}
\bar{r}_{1}-\bar{a} \exp x_{1}-\bar{b} \exp x_{2} \\
\bar{r}_{2}-\bar{c} \exp x_{1}-\bar{d} \exp x_{2}
\end{array}\right] \neq\left[\begin{array}{l}
0 \\
0
\end{array}\right] .
$$

Furthermore, it can easily be seen that

$$
\operatorname{deg}\left\{J Q N\left(x_{1}, x_{2}\right)^{T}, \Omega \cap \operatorname{Ker} L,(0,0)^{T}\right\}=\operatorname{sign}\left[(\bar{a} \bar{d}-\bar{b} \bar{c}) v_{1}^{*} v_{2}^{*}\right] \neq 0,
$$

where $J$ is the identity mapping since $\operatorname{Im} Q=\operatorname{Ker} L$. By now we know that $\Omega$ verifies all the requirements of Lemma 2.1, and then (2.12) has at least one $\omega$-periodic solution. The proof is complete. 
In view of Theorem 2.3, we can easily obtain the following corollary.

COROLLARY 2.4. Assume that Theorem 2.3(i) and (ii) hold and that

$$
\bar{r}_{1} \bar{d}-\bar{r}_{2} \bar{b}>0, \quad \overline{a r}_{2}-\overline{c r}_{1}>0, \quad \bar{a} \bar{d}-\bar{b} \bar{c}>0 .
$$

Then system (1.3) has at least one positive $\omega$-periodic solution.

REMARK 2.5. The continuous counterpart of (1.3) under the assumption of Corollary 2.4 is

$$
\begin{aligned}
& u^{\prime}(t)=u(t)\left[r_{1}(t)-a(t) u\left(t-n_{1}\right)-b(t) v\left(t-n_{2}\right)\right] \\
& v^{\prime}(t)=v(t)\left[r_{2}(t)-c(t) u\left(t-l_{1}\right)-d(t) v\left(t-l_{2}\right)\right]
\end{aligned}
$$

where $r_{1}(t), a(t), b(t), r_{2}(t), c(t)$, and $d(t)$ are positive periodic continuous functions with period $\omega>0$, and $\tau(t), \sigma(t)$ are periodic continuous functions with period $\omega>0$. Huo and Li [13] have shown that if

$$
\begin{gathered}
\bar{r}_{1}-\bar{b} \frac{\bar{r}_{1}}{\bar{a}} \exp 2 \bar{r}_{2} \omega>0, \quad \bar{r}_{2}-\bar{c} \frac{\bar{r}_{1}}{\bar{b}} \exp 2 \bar{r}_{1} \omega>0, \\
\bar{r}_{1} \bar{d}-\bar{r}_{2} \bar{b}>0, \quad \overline{a r}_{2}-\overline{c r}_{1}>0, \quad \bar{a} \bar{d}-\bar{b} \bar{c}>0,
\end{gathered}
$$

where

$$
\bar{f}=\frac{1}{\omega} \int_{0}^{\omega} f(t) d t
$$

then (2.41) has a positive periodic solution. Clearly, the criteria established above are similar to those for corresponding continuous systems.

REMARK 2.6. Theorem 2.3 remains valid if some or all terms are replaced by terms with variable delays.

3. Global stability of positive periodic solutions. In this section, we derive sufficient conditions which guarantee that the positive $\omega$-periodic solution of (1.3) is globally stable. Our strategy in the proof of the global stability of the positive $\omega$-periodic solution of (1.3) is to construct suitable Lyapunov functions

THEOREM 3.1. In addition to the assumptions in Theorem 2.3, assume further that

(i) there exist positive constant $v$ and positive constants $c_{i}$ such that for all large $k$,

$$
\min \left\{c_{1} a(k)-c_{2} c(k), c_{2} d(k)-c_{1} d(k)\right\}>v,
$$


(ii) $a(k) \beta_{1} \leq 1, d(k) \beta_{2} \leq 1$, for all large $k$, where $\beta_{i}$ are given in Theorem 2.3,

(iii) $l_{i}=0, n_{i}=0, i=1,2$.

Then the positive $\omega$-periodic solution of (1.3) is globally stable.

Proof. The existence of the positive $\omega$-periodic solution of (1.3) follows from Theorem 2.3. Let $\left\{u^{*}(k), v^{*}(k)\right\}$ be a positive $\omega$-periodic solution of (1.3). We prove below that it is uniformly asymptotically stable. To this end, we introduce the change of variables

$$
u_{1}(k)=u(k)-u^{*}(k), \quad u_{2}(k)=v(k)-v^{*}(k) .
$$

System (1.3) is then transformed into

$$
\begin{aligned}
u_{1}(k+1)= & u(k) \exp \left[r_{1}(k)-a(k) u(k)-b(k) v(k)\right] \\
& -u^{*}(k) \exp \left[r_{1}(k)-a(k) u^{*}(k)-b(k) v^{*}(k)\right], \\
u_{2}(k+1)= & v(k) \exp \left[r_{2}(k)-c(k) u(k)-d(k) v(k)\right] \\
& -v^{*}(k) \exp \left[r_{2}(k)-c(k) u^{*}(k)-d(k) v^{*}(k)\right],
\end{aligned}
$$

which can be rewritten as

$$
\begin{aligned}
u_{1}(k+1)= & \exp \left[r_{1}(k)-a(k) u^{*}(k)-b(k) v^{*}(k)\right] \\
& \times\left(\left(1-a(k) u^{*}(k)\right) u_{1}(k)-b(k) u^{*}(k) u_{2}(k)+f_{1}(k, u(k))\right), \\
u_{2}(k+1)= & \exp \left[r_{2}(k)-c(k) u^{*}(k)-d(k) v^{*}(k)\right] \\
& \times\left(\left(1-d(k) v^{*}(k)\right) u_{2}(k)+c(k) v^{*}(k) u_{1}(k)+f_{2}(k, u(k))\right),
\end{aligned}
$$

where $\left|f_{i}(k, u)\right| /\|u\|$ converges, uniformly with respect to $k \in \mathbb{N}$, to zero as $\|u\| \rightarrow 0$. In view of system (1.3), it follows from (3.4) that

$$
\begin{aligned}
& u_{1}(k+1) \\
& =u^{*}(k+1)\left(\left(1-a(k) u^{*}(k)\right) \frac{u_{1}(k)}{u^{*}(k)}-b(k) u_{2}(k)+\frac{f_{1}(k, u(k))}{u^{*}(k)}\right), \\
& u_{2}(k+1) \\
& =v^{*}(k+1)\left(\left(1-d(k) v^{*}(k)\right) \frac{u_{2}(k)}{v^{*}(k)}+c(k) u_{1}(k)+\frac{f_{2}(k, u(k))}{v^{*}(k)}\right) .
\end{aligned}
$$

We define the function $V$ by

$$
V(u(k))=c_{1}\left|\frac{u_{1}(k)}{u^{*}(k)}\right|+c_{2}\left|\frac{u_{2}(k)}{v^{*}(k)}\right|
$$


where $c_{j}$ are positive constants given in (i). Calculating the difference of $V$ along the solution of system (3.5) and using (ii), we obtain

$$
\begin{aligned}
\Delta V \leq & -\left(c_{1} a(k)-c_{2} c(k)\right) x_{1}^{*}(k)\left|\frac{u_{1}(k)}{x_{1}^{*}(k)}\right| \\
& -\left(c_{2} d(k)-c_{1} b(k)\right) x_{2}^{*}(k)\left|\frac{u_{2}(k)}{x_{2}^{*}(k)}\right| \\
& +c_{1} \frac{\left|f_{1}(k, u(k))\right|}{u^{*}(k)}+c_{2} \frac{\left|f_{2}(k, u(k))\right|}{u^{*}(k)} .
\end{aligned}
$$

Since $\left|f_{i}(k, u)\right| /\|u\|$ converges uniformly to zero as $\|u\| \rightarrow 0$, it follows from condition (i) and Theorem 2.3 that there is a positive constant $\gamma$ such that if $k$ is sufficiently large and $\|u(k)\|<\gamma$,

$$
\Delta V \leq-\frac{v\|u(k)\|}{2}
$$

By [1], we see that the trivial solution of (3.5) is uniformly asymptotically stable, and so is the solution $\left\{u^{*}(k), v^{*}(k)\right\}$ of (1.3). Note that the positive solution $\{u(k), v(k)\}$ is chosen in an arbitrary way. Proceeding exactly as in [24], we conclude that the positive periodic solution $\left\{u^{*}(k), v^{*}(k)\right\}$ of (1.3) is globally stable. The proof is complete.

REMARK 3.2. Theorem 3.1 indicates that diagonally dominant interaction matrices, together with small growth rates and strong persistence, ensure that the positive periodic solution of system (1.3) is globally asymptotically stable.

REMARK 3.3. There are still many interesting and challenging mathematical questions to be studied for system (1.3). For example, we did not discuss the bifurcations that occur when conditions of stability are violated. We will leave this for future work.

AcKNOWLEDgments. This work is supported by the National Natural Science Foundation (NNSF) of China (10171040), the National Science Foundation (NSF) of Gansu Province of China (ZS011-A25-007-Z), and the Foundation for University Key Teacher by the Ministry of Education of China. It is also supported by the Teaching and Research Award Program for Outstanding Young Teachers in Higher Education Institutions of Ministry of Education of China, the Key Research and Development Program for Outstanding Groups of Gansu University of Technology, and the Development Program for Outstanding Young Teachers in Gansu University of Technology.

\section{REFERENCES}

[1] R. P. Agarwal, Difference Equations and Inequalities: Theory, Methods, and Applications, Monographs and Textbooks in Pure and Applied Mathematics, vol. 228, Marcel Dekker, New York, 2000. 
[2] R. P. Agarwal and P. J. Y. Wong, Advanced Topics in Difference Equations, Mathematics and Its Applications, vol. 404, Kluwer Academic, Dordrecht, 1997.

[3] S. Ahmad and A. C. Lazer, Necessary and sufficient average growth in a LotkaVolterra system, Nonlinear Anal. 34 (1998), no. 2, 191-228.

[4] _ Average conditions for global asymptotic stability in a nonautonomous Lotka-Volterra system, Nonlinear Anal., Ser. A: Theory Methods 40 (2000), no. 1-8, 37-49.

[5] M. Fan and K. Wang, Periodic solutions of a discrete time nonautonomous ratiodependent predator-prey system, Math. Comput. Modelling 35 (2002), no. 9-10, 951-961.

[6] J. E. Franke and A.-A. Yakubu, Mutual exclusion versus coexistence for discrete competitive systems, J. Math. Biol. 30 (1991), no. 2, 161-168.

[7] _ Geometry of exclusion principles in discrete systems, J. Math. Anal. Appl. 168 (1992), no. 2, 385-400.

[8] - Species extinction using geometry of level surfaces, Nonlinear Anal. 21 (1993), no. 5, 369-378.

[9] H. I. Freedman, Deterministic Mathematical Models in Population Ecology, Monographs and Textbooks in Pure and Applied Mathematics, vol. 57, Marcel Dekker, New York, 1980.

[10] R. E. Gaines and J. L. Mawhin, Coincidence Degree, and Nonlinear Differential Equations, Springer-Verlag, Berlin, 1977.

[11] K. Gopalsamy, Global asymptotic stability in a periodic Lotka-Volterra system, J. Austral. Math. Soc. Ser. B 27 (1985), no. 1, 66-72.

[12] J. Hofbauer, V. Hutson, and W. Jansen, Coexistence for systems governed by difference equations of Lotka-Volterra type, J. Math. Biol. 25 (1987), no. 5, 553-570.

[13] H.-F. Huo and W.-T. Li, Periodic solution of a periodic two-species competition model with delays, to appear in Int. J. Appl. Math.

[14] _ Periodic solutions of a ratio-dependent food chain model with delays, to appear in Taiwanese J. Math.

[15] H.-F. Huo, W.-T. Li, and S. S. Cheng, Periodic solutions of two-species diffusion models with continuous time delays, Demonstratio Math. 35 (2002), no. 2, 433-446.

[16] Y. Kuang, Delay Differential Equations with Applications in Population Dynamics, Mathematics in Science and Engineering, vol. 191, Academic Press, Massachusetts, 1993.

[17] J. D. Murray, Mathematical Biology, Biomathematics, vol. 19, Springer-Verlag, Berlin, 1989.

[18] A. J. Nicholson, The balance of animal populations, J. Animal Ecology 2 (1933), 132-178.

[19] S. G. Ruan and X.-Z. He, Global stability in chemostat-type competition models with nutrient recycling, SIAM J. Appl. Math. 58 (1998), no. 1, 170-192.

[20] Y. Saito, W. Ma, and T. Hara, A necessary and sufficient condition for permanence of a Lotka-Volterra discrete system with delays, J. Math. Anal. Appl. 256 (2001), no. 1, 162-174.

[21] S. H. Saker and S. Agarwal, Oscillation and global attractivity in a nonlinear delay periodic model of respiratory dynamics, Comput. Math. Appl. 44 (2002), no. 5-6, 623-632.

[22] - Oscillation and global attractivity in a periodic Nicholson's blowflies model, Math. Comput. Modelling 35 (2002), no. 7-8, 719-731. 
[23] L. L. Wang and W.-T. Li, Existence and global stability of positive periodic solutions of a predator-prey system with delays, to appear in Appl. Math. Comput.

[24] W. D. Wang and Z. Y. Lu, Global stability of discrete models of Lotka-Volterra type, Nonlinear Anal., Ser. B: Real World Applications 35 (1999), no. 8, 10191030.

[25] J. Yan and Q. Feng, Global attractivity and oscillation in a nonlinear delay equation, Nonlinear Anal. 43 (2001), no. 1, 101-108.

Hai-Feng Huo: Department of Applied Mathematics, Gansu University of Technology, Lanzhou, Gansu 730050, China

Wan-Tong Li: Department of Mathematics, Lanzhou University, Lanzhou, Gansu 730000, China

E-mail address: wt1i@1zu.edu.cn 


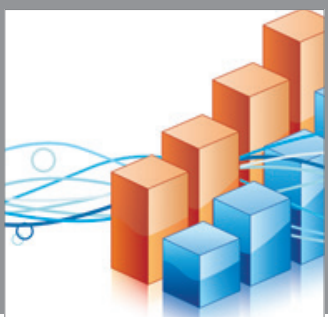

Advances in

Operations Research

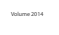

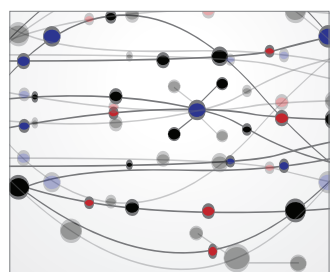

\section{The Scientific} World Journal
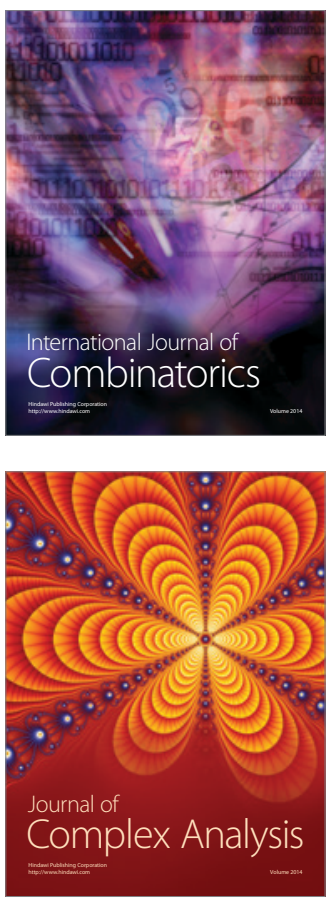

International Journal of

Mathematics and

Mathematical

Sciences
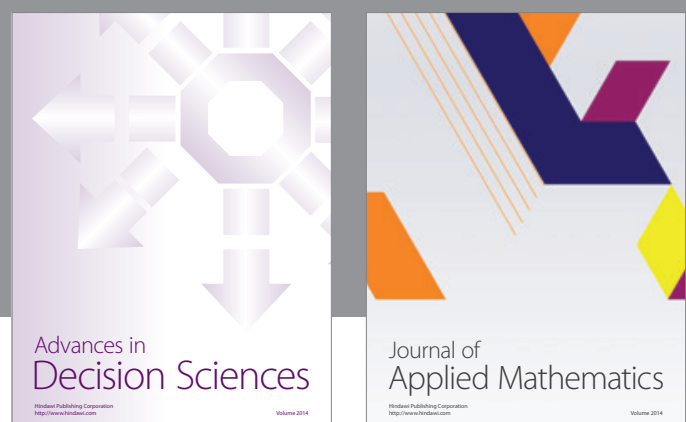

Journal of

Applied Mathematics
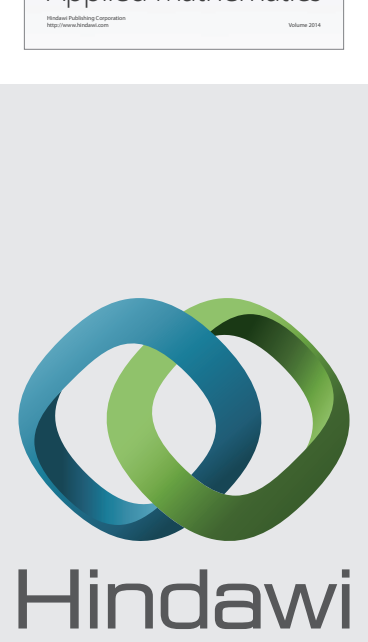

Submit your manuscripts at http://www.hindawi.com
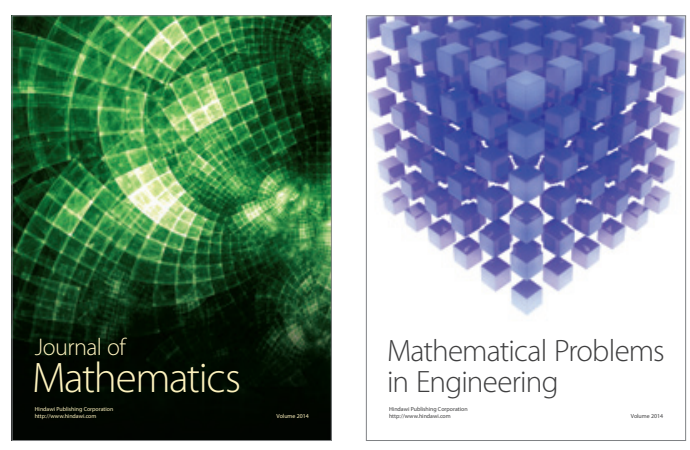

Mathematical Problems in Engineering
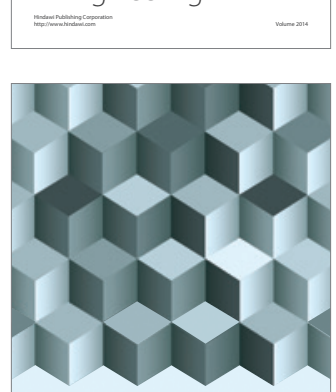

Journal of

Function Spaces
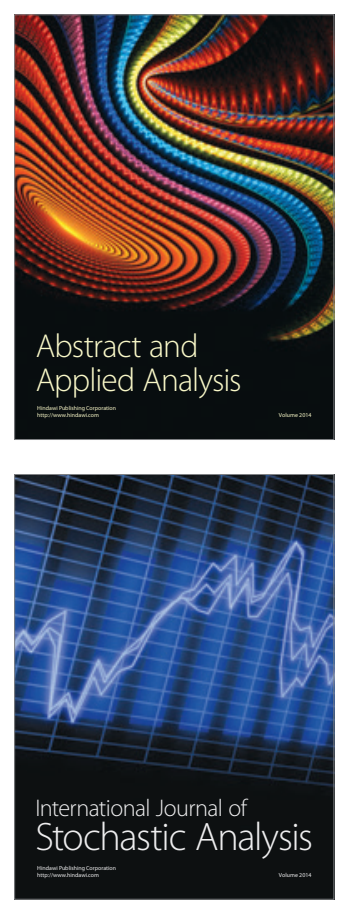

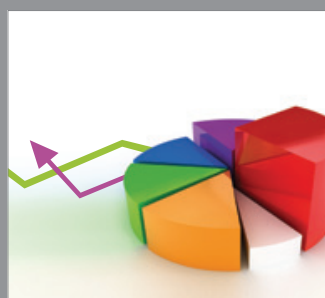

ournal of

Probability and Statistics

Promensencen
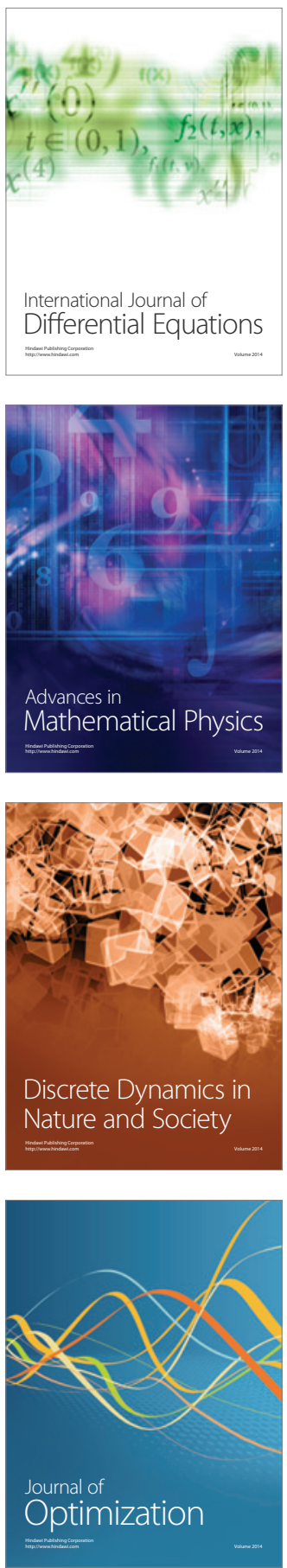